\title{
Lasers in Fixed Prosthodontics
}

\section{El khourani Wadie ${ }^{1 *}$ and Pr Amal El yamani ${ }^{2}$}

${ }^{1}$ Resident in Prosthodontics Department, Mohamed V University, Faculty of Dental Medicine of Rabat

${ }^{2}$ Associate Professor, Head of the Department of Prosthodontics Mohamed V University, Faculty of Dental Medicine of Rabat

*Corresponding Author: El khourani Wadie, Resident in Prosthodontics Department, Mohamed V University, Faculty of Dental Medicine of Rabat.

Received: October 15, 2019; Published: November 21, 2019

DOI: $10.31080 /$ ASDS.2019.03.0707

\begin{abstract}
Among the most significant events in the evolution of modern prosthodontics is the introduction of lasers. A laser (acronym for light amplification by stimulated emission of radiation) is a device that produces radiation which is spatially and temporally coherent.

In fixed prostheses, lasers find their indications in the management of soft and hard tissues, they are characterized by their precision, their speed as well as the positive response of the tissues treated by this technique in comparison with other surgical techniques.

This work will describe the different types of lasers, their uses in fixed prosthodontics, their indications and their interactions with the tissues concerned.
\end{abstract}

Keywords: Lasers; Prosthodontics

\section{Introduction}

One of the most significant events in the evolution of modern fixed prosthodontics is the introduction of lasers. A laser (acronym for light amplification by stimulated emission of radiation) is a device that produces radiation that is spatially and temporally coherent [1].

Currently the laser has become an integral part of a complete treatment plan. Since its first application in 1960 in the field of dentistry, its current use has replaced several technical and surgical steps [2].

The purpose of this work is to synthesize the fundamental principles of lasers and their applications in the various fields of fixed prosthodontics.

\section{Invention of the laser}

The theory presented by Einstein in 1917 on stimulated emissions can be considered as the founding element of laser studies.40 years later, the American physicist Charles Townes developed Einstein's theory thanks to the principle of amplifying microwave frequencies by stimulated emission who takes the acronym maser [3].

The appearance of the first functional laser was in 1967, the ruby laser signed by T. Maiman, in 1961, Snitzer introduced the neodymium laser.

In 1962, the first medical laser was developed in the laboratories of the University of Cincinnati following the work of Goldman, who is the first doctor to have used the laser in the medical field.

Keifhabes., et al. were the first to use the Nd: yttrium-aluminumgarnet (YAG) laser.

In 1988 Francis l'Esperance was the first to use the argon laser in ophthalmology [4].

In 1990 the first dental laser was released. 


\section{Laser classification}

The different laser systems used in prosthodontics can be classified into several categories according to their wavelengths:

Laser erbium

The erbium laser family has two wavelengths, the YSGG laser (yttrium scandium gallium garnet, $2780 \mathrm{~nm}$ ) and the ER laser, YAG (yttrium aluminium garnet $2940 \mathrm{~nm}$ ).

The ER laser, YSGG, has a solid crystal of yttrium scandium gallium garnet doped with erbium and chromium. The ER laser, YAG, has a solid crystal of erbium-doped yttrium aluminum garnet [5].

The wavelengths of erbium have a high affinity for hydroxyapatite, as well as a remarkable water absorption capacity compared to other lasers, which implies that erbium's are the lasers of choice for dental hard tissue treatments [6] and soft tissue procedures (high presence of $\mathrm{H}_{2} \mathrm{O}$ in soft tissues) [7].

$\mathrm{CO}_{2}$ laser

The $\mathrm{CO}_{2}$ laser has an active gaseous environment who is pumped through the electrical discharge current. The wavelength of these lasers is $10,600 \mathrm{~nm}$.

Due to its cutting capacity, coagulating power and short tissue penetration depth, $\mathrm{CO}_{2}$ lasers are indicated for the treatment of mucosal lesions and the vaporization of dense fibrous tissues [8].

Argon laser

The argon laser system uses argon as the active environment. Argon lasers have two wavelengths used in dentistry: $488 \mathrm{~nm}$ (blue) and $514 \mathrm{~nm}$ (blue-green) in the visible light spectrum. The $488 \mathrm{~nm}$ wavelength is used for photopolymerization of composite restorative materials, activation of bleaching gels and detection of dental cavities [9].

The advantages of using the argon photopolymerization system are the improved physical properties of composite resins as well as reducing time for curing compared to the ordinary blue light.

The $514 \mathrm{~nm}$ wavelength is used for soft tissue surgeries such as acute inflammatory periodontal disease and highly vascularized lesions, such as hemangiomas due to its maximum absorption into tissues containing hemoglobin, hemosiderin and melanin [10].
Neodymium laser

Neodymium laser Yttrium Aluminium Garnet: Nd: YAG has a solid active environment, which is a garnet crystal combined with rare earth elements (yttrium and aluminium), doped with neodymium ions.

The wavelength Nd: YAG is strongly absorbed by the pigmented tissue, making it a very useful surgical instrument for cutting and coagulating soft tissues with good hemostasis. The available dental models have an emission wavelength of $1064 \mathrm{~nm}$, which is located in the invisible part of the near infrared of the electromagnetic spectrum. In addition to its surgical applications, $\mathrm{Nd}$ : YAG is also indicated for the non-surgical debridement of periodontal disease.

\section{Laser diode}

The diode laser is a solid-state semiconductor laser that uses a combination of gallium, arsenide, aluminum and indium. The wavelengths used in dentistry are between 800 and $980 \mathrm{~nm}$ and are poorly absorbed in water, unlike hemoglobin and other pigments where they are highly absorbed. This laser is excellent for soft tissue interventions because it does not interact with dental and bone hard tissue. diode lasers are also used as adjuvant therapy for the bio-stimulation of osteoblasts around implants [11].

\section{Application of the laser in fixed prosthodontics}

\section{Coronary elongation}

Lasers offer the operator precision, comfort and control of the surgical procedure that are far superior to other surgical techniques. Lasers allow the incision lines to be finely drawn and the desired gingival contour to be formed. All other coronary elongation methods have disadvantages in the surgical approach, the healing time is longer, the position of the gingival margin after healing is random and the postoperative outcomes are uncomfortable and painful for the patient. In electrosurgery, the electric scalpel emits significant heat that can cause pulp and bone necrosis [12].

Er: YAG lasers have a high potential for bone ablation due to the mineralized matrix of bone that contains water and hydroxyapatite (very high absorption rate) [13].

Any technique used for coronary elongation in the anterior area should ensure that an optimal esthetic result is obtained, hence the interest of using lasers. 
The laser coronal elongation is indicated in:

- The presence of subgingival cavities.

- Coronary fracture that extends apically below the marginal gumline.

- Endodontic perforation close to the alveolar rim.

- Insufficiency of the coronary lenght.

- Difficult positioning of the finishing line at a distance from the biological space.

- Disordered gingival architecture.

Soft tissue management around the abutments

The energy supplied by the argon laser has a peak absorption in hemoglobin, thus ensuring excellent hemostasis and well-regulated vaporization of oral tissues. These characteristics are beneficial for the retraction and hemostasis of gingival tissue around peripheral preparations before an impression is taken.

The argon laser used for gingival eviction must have a $300 \mathrm{um}$ fiber, a power setting of 1.0 W and a continuous wave emission [14].

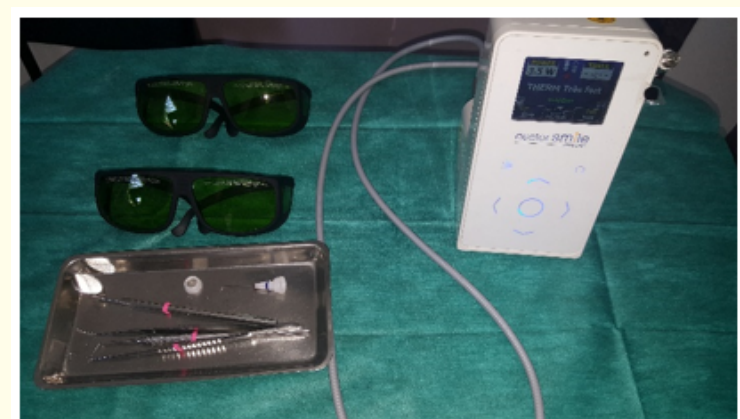

Figure 1: Technical tray for laser gingival eviction containing a diode laser, protective glasses for practitioner and patient and examination tray.

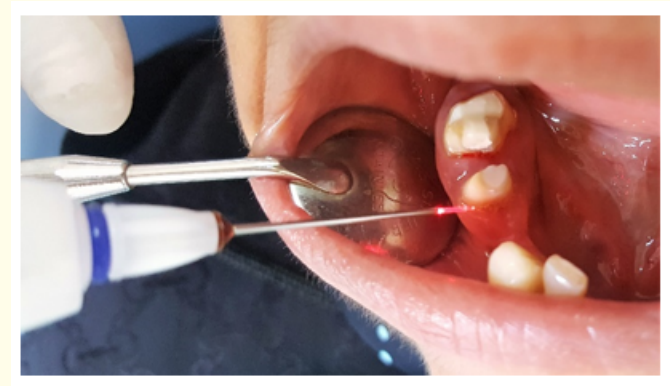

Figure 2: Gingival evacuation by laser diode around the prepared stump of the 45 before the impression is taken, the fiber is placed in the sulcus in contact with the tissue. With a sweeping motion, the fiber is moved around the tooth under a spray of water spray associated with a suction.

\section{Formation of oval pontic sites}

Dental avulsion causes alveolar resorption of edentulous sites. After the healing is complete, a bone ridge covered with a flat fibromucosa will form.

This architecture is considered inappropriate for plural restoration, especially in the anterior maxillary areas, as it leads to an unaesthetic design of the pontic elements associated with phonation and hygiene problems.

For a good design of the pontic sites, the use of lasers can be indicated for soft tissue and bone remodeling. Soft tissue surgery can be performed with argon and $\mathrm{CO}_{2}$ lasers and bone surgery can be performed with the Erbium laser family.

\section{Removal of ceramic veneers}

The use of lasers when removing veneers allows the practitioner to avoid cutting out the bonded elements, it also improves the patient's comfort and reduces the time in chair-side.

The energy emitted by the laser passes through the ceramic while remaining unchanged, then absorbed by the water molecules present in the bonding agent.

The detachment is made at the junction of the silane and the resin, the underlying tooth does not suffer any trauma during this operation [15].

Root canal etching during Direct fiber-reinforced composit restoration

Adhesion is an essential element for the success of Direct fiberreinforced composite restoration. it is generally impossible to create a bonding surface without the presence of smear layer. This structure contains hydroxyapatite, destroyed collagen fibers and other elements that can alter the quality of the adhesion.

Er: YAG lasers cause thermomechanical ablation by microexplosions. This type of laser evaporates water and organic components. The pressure interval during this process increases by causing micro-explosions in the inorganic structures leading to the opening of the dentine tubules [16].

The resulting irregularity improves the adhesive properties of the dentine surface, ensuring then a better bonding.

\section{Lasers in implantology}

Dental lasers also find their indications in implantology, from the preparation of the implant site before placement, to the biostimulation of peri-implant tissues after osteointegration of the implant and finally as a therapy for peri-impactites.

One of the advantages of using lasers in implantology is the ease of intervention on peri-implant soft tissues, particularly in the 2 nd surgical stage due to its hemostatic effect and the minimal 
retraction of peri-implant soft tissues after laser surgery, which ensures that tissue margins remain at the same level after healing as they are immediately after surgery [17].

Preparation of the implant site: Lasers can be used for the placement of mini-implants, especially in patients with bleeding problems, in order to perform bone surgery without blood diffusion.

Treatment of peri-implantitis: Diode lasers, $\mathrm{CO}_{2}$ lasers and Er: YAG lasers have been used for the treatment of peri-implant diseases, due to their bactericidal effect and their technical simplicity.

Debridement of the abutment-implant interface with lasers can effectively decontaminate surfaces, reduce the number of bacteria and improve the success rate of contaminated implant Schwarz., et al. have demonstrated the effectiveness of Er: YAG laser treatment to eliminate subgingival calculation of titanium implant surfaces without causing any thermal damage $[18,19]$.

Nd: YAG and Ho: YAG lasers are not suitable for the treatment of peri-impactites and cause fusion, loss of porosity and other surface alterations [20].

\section{Conclusion}

The use of different types of lasers in prosthodontics has made it possible to redefine the principles of soft and hard tissue management around dental and implant abutments, offering the practitioner the possibility of performing Multiple procedures in complete comfort and with great predictability of results.

However, the dentist's responsibility requires him to choose the right laser, the right wavelength and the lowest possible amount of energy to achieve the desired results.

\section{Bibliography}

1. L Walsh. "The current status of laser applications in dentistry”. Australian Dental Journal 48 (2003): 146-155.

2. G Kesler. "Clinical applications of lasers during removable prosthetic reconstruction". Dental Clinics of North America 48 (2004): 963-969.

3. AM Bhat. "Lasers in prosthodontics-An overview part 1: Fundamentals of dental lasers". The Journal of Indian Prosthodontic Society 10 (2010): 13-26.
4. S Kaura., et al. "Lasers in prosthodontics". The Saint's International Dental Journal 1 (2015) 11.

5. G van As. "Erbium lasers in dentistry". Dental Clinics of North America 48 (2004): 1017-1059.

6. T Harashima., et al. "Morphological comparative study on ablation of dental hard tissues at cavity preparation by Er: YAG and Er, Cr: YSGG lasers". Photomedicine and Laser Therapy 23 (2005): 52-55.

7. I Ishikawa., et al. "Clinical application of erbium: YAG laser in periodontology". Journal of the International Academy of Periodontology 10 (2008): 22-30.

8. M Pogrel., et al. "Structural changes in dental enamel induced by high energy continuous wave carbon dioxide laser". Lasers in Surgery and Medicine 13 (1993): 89-96.

9. MG Fleming., et al. "Photopolymerization of composite resin using the argon laser". Journal-Canadian Dental Association 65 (1999): 447-452.

10. DJ Coluzzi. "Fundamentals of dental lasers: science and instruments". Dental Clinics of North America 48 (2004): 751-770.

11. O Dortbudak., et al. "Biostimulation of bone marrow cells with a diode soft laser". Clinical Oral Implants Research 11 (2000): 540-545.

12. S Parker. "The use of lasers in fixed prosthodontics". Dental Clinics of North America 48 (2004): 971-998.

13. JR Jyothy., et al. "Lasers in prosthetic dentistry". Indian Journal of Applied Research 3 (2013): 369-370.

14. V Punia., et al. "The current status of laser applications in". Prosthodontics (2012).

15. U Iseri., et al. "Effect of Er:YAG laser on debonding strength of laminate veneers". European Journal of Dentistry 8 (2014): 58-62.

16. O Parlar Oz., et al. "Effect of Laser Etching on Glass Fiber Posts Cemented with Different Adhesive Systems". Photomedicine and Laser Surgery 36.1 (2017): 51-57.

17. GE Romanos., et al. "Lasers use in dental implantology". Implant Dentistry 22 (2013): 282-288.

18. RA Strauss. "Lasers in oral and maxillofacial surgery". Dental Clinics of North America 44 (2000): 851-873. 
19. T Kato., et al. "Bactericidal efficacy of carbon dioxide laser against bacteria-contaminated titanium implant and subsequent cellular adhesion to irradiated area". Lasers in Surgery and Medicine 23 (1998): 299-309.

20. M Kreisler., et al. "Effect of Nd: YAG, Ho: YAG, Er: YAG, CO 2, and GaAlAs Laser Irradiation on Surface Properties of Endosseous Dental Implants". International Journal of Oral and Maxillofacial Implants 17.2 (2002): 202-211.

Volume 3 Issue 12 December 2019

(C) All rights are reserved by El khourani Wadie and

Pr Amal El yamani. 